\title{
Efficacy of Zhifei Kangfu Decoction in the Treatment of Infantile Pneumonia in the Recovery Period
}

\author{
Yingying Zhang ${ }^{1, \mathrm{a}}$, Caihong $\mathrm{Cao}^{2, \mathrm{~b}}, \mathrm{Xin}_{\mathrm{Guo}}^{3, \mathrm{c}}$, Haijun Shan ${ }^{4, \mathrm{~d}}$ (corresponding author) \\ 1,2,3,4 Henan Province Hospital of Traditional Chinese Medicine Zhengzhou Henan 450000
}

\begin{abstract}
The objective of this article is to explore the effect of applying Zhifei Kangfu Decoction on the treatment effect of patients during the recovery period of children with pneumonia. The method of this research is to take patients who were treated in our hospital from December 2019 to December 2020 as an example to carry out the research work. The researchers selected all patients in the recovery period of pediatric pneumonia, and the number was selected as 100 cases, who were divided into two groups, and the treatment methods used are conventional western medicine and Zhifei Kangfu Decoction treatment, who were named the control group and the experimental group, and the clinical treatment effects of the two groups of patients are compared and analyzed. The effective rate and adverse reaction rate of children in the experimental group were $96.00 \%$ and $4.00 \%$, respectively. The effective rate and adverse reaction rate of children in the control group were $82.00 \%$ and $30.00 \%$, respectively. Asthma, cough relieving, and treatment time were shorter than those of the control group, and the difference in the data was $\mathrm{P}<0.05$, which was statistically significant. The experimental group had better results. The treatment of children in the recovery period of pneumonia and the application of Zhifei Kangfu Decoction can promote the improvement of clinical efficacy, reduce the incidence of adverse reactions in children, and have a positive significance in promoting the recovery of children.
\end{abstract}

\section{Introduction}

Pediatric pneumonia has a high clinical incidence, and is a common disease in pediatrics, which poses a great threat to the health of patients[1]. For most children with pneumonia, the clinical treatment is to apply antibiotics for intravenous anti-inflammatory methods, and to relieve cough and asthma, improve the clinical symptoms of the children, and then improve the patient's cough, expectoration and other symptoms[2] after the patient enters the recovery period. In this process, if antibiotics continue to be used, not only will it not improve the patient's condition, but it will also easily lead to adverse reactions such as drug resistance and flora imbalance in the patient[3]. On this basis, this study applied Zhifei Kangfu Decoction to explore its effect on the recovery period of children with pneumonia. The analysis is as follows.

\section{Target}

Through the use of different treatment methods to compare, explore the effect of Zhifei Kangfu Decoction on the recovery effect of children with pneumonia in the recovery period, and judge the clinical application value of the treatment methods.

\section{Methods}

\subsection{General information of patients}

The selected research site is our hospital, and the research period is from December 2019 to December 2020. The patient selection criteria is the recovery of pediatric pneumonia, and the number is 100 . Patients were divided into two groups with 50 people in each group for group treatment, named the control group and the experimental group with conventional western medicine treatment and Zhifei Kangfu decoction treatment respectively. In the experimental group, the maximum and minimum age of patients were 7 years and 2 years old respectively, and the average age of the patients was $(4.34 \pm 0.33)$ years old. In the control group, the maximum and minimum age of patients were 7 years and 3 years respectively, and the average age of patients was $(4.98 \pm 0.56)$ years. The general data of the two groups of children were compared, and the results indicated that $\mathrm{P}>0.05$, which was comparable.

Inclusion criteria: The patient meets the clinical diagnostic criteria for pneumonia in children in "Practical Pediatrics", with symptoms such as cough, fever, and wheezing; the physical characteristics of the lungs are more obvious, and dense small and medium blisters can be seen on auscultation of both lungs; Under X-ray examination, the lungs of the children have obvious 
positive alterations. After antibiotics were given to the children, the children's body temperature returned to normal, the cough was relieved, and small blisters were not heard in the lungs, but there were still rales or phlegm sounds, and the flaky shadows changed to lung texture, and the children entered recovery period. The family members of the children in this study were informed of the experimental process and agreed to participate. The study was implemented after the approval of the hospital ethics committee.

Exclusion criteria: children with mental disorders; children with other major diseases; children with poor compliance and withdrew from the study halfway.

\subsection{Research methods}

The children in the control group were given conventional western medicine treatment, mainly using amoxicillin granules. The medication should be calculated based on the actual situation of the children, combined with the age and weight of the children, and the drug was taken orally, with intervention 3 times a day.

The treatment method for children in the experimental group is Zhifei Kangfu Decoction. The drugs mainly include: orange, red peony, aster, fritillaria, coltsfoot, licorice, cabbage seed, peach kernel and turmeric. Decoction of all medicines, the dosage of the drug was determined according to the actual situation of the children with one dose per day.

\subsection{Observation indicators}

Treatment indicators: Compare the disappearance time of symptoms and signs such as fever, cough, sputum, and lung rales in the two groups.

Therapeutic effect: It mainly includes three indicators of cured, improved and ineffective. Cured means that the children's body temperature returns to normal after receiving therapeutic intervention, and the cough symptoms disappeared. The breath sounds of the lungs of the children are auscultated, which is relatively clear, or slightly thicker with inconspicuous rale symptom. Improved means that the children's body temperature has basically returned to normal after the treatment intervention, the children's cough symptoms have been relieved, and there is sputum, but the auscultation rales are significantly reduced. Ineffective means that the children's clinical symptoms did not improve significantly before and after treatment. Excluding inefficiency is the total effective rate of this study.

Adverse reactions: The adverse reactions of the two groups of patients were observed and analyzed, which mainly include drug resistance, rash, nausea and vomiting.

\subsection{Statistical methods}

Use the statistical software SPSS20.0 as a tool to perform statistical analysis on the data appearing in this study, verifying the comparison results of the measurement data $(\mathrm{x} \pm \mathrm{s})$ with the $\mathrm{t}$ value, and verify and compare the count data $(n, \%)$ by the $\mathrm{X}^{2}$ value. When the results showed $\mathrm{P}<0.05$, it indicated that the differences between the groups had statistical analysis value.

\section{Result}

\subsection{Disappearance time of clinical symptoms}

Compared with the control group, the experimental group had shorter cough, anti-asthmatic, rale absorption time than the control group, and the treatment time was relatively short. There was a big difference between the groups, $\mathrm{P}<0.05$, which was statistically significant.

Table1. Comparison of disappearance time of various clinical symptoms ( $\mathrm{x} \pm \mathrm{s})$

\begin{tabular}{ccccc}
\hline Group & shorter cough & anti-asthmatic & rale absorption & $\begin{array}{c}\text { treatment } \\
\text { timeshorter cough }\end{array}$ \\
\hline $\begin{array}{c}\text { Experimental group } \\
(\mathbf{n = 5 0})\end{array}$ & $5.34 \pm 1.76$ & $3.62 \pm 0.66$ & $4.87 \pm 0.87$ & $5.65 \pm 1.33$ \\
$\begin{array}{c}\text { Control group } \\
(\mathbf{n = 5 0})\end{array}$ & $7.00 \pm 1.76$ & $4.65 \pm 1.43$ & $5.64 \pm 1.43$ & $8.65 \pm 1.23$ \\
$\mathbf{t}$ & 7.596 & 9.556 & 4.335 & 12.346 \\
$\mathbf{P}$ & $<0.05$ & $<0.05$ & $<0.05$ & $<0.05$ \\
\hline
\end{tabular}

\subsection{Therapeutic effect}

In the experimental group, 48 children indicated that the treatment was effective, with an effective rate of $96.00 \%$, and 41 children in the control group indicated that the treatment was effective, with an effective rate of $82.00 \%$. The difference in data was statistically significant $(\mathrm{P}<0.05)$.

Table2. Comparison of effective treatment rates between the two groups of patients [n(\%)]

\begin{tabular}{lllll}
\hline Group & $\begin{array}{l}\text { Marked } \\
\text { effective }\end{array}$ & Effective & Ineffective & Effective rate \\
\hline
\end{tabular}




\begin{tabular}{ccccc}
\hline $\begin{array}{c}\text { Experimental } \\
\text { group }(\mathbf{n = 5 0})\end{array}$ & $35(70.00)$ & $13(26.00)$ & $2(4.00)$ & $48(96.00)$ \\
$\begin{array}{c}\text { Conrol group } \\
(\mathbf{n}=\mathbf{5 0})\end{array}$ & $24(48.00)$ & $17(34.00)$ & $9(18.00)$ & $41(82.00)$ \\
$\mathbf{X 2}$ & & & 8.996 \\
$\mathbf{P}$ & & & $<0.05$ \\
\hline
\end{tabular}

\subsection{Incidence of adverse reactions}

The incidence of adverse reactions in the experimental group was $4.00 \%$, which was significantly lower than the $30.00 \%$ in the control group. The difference in data between the two groups indicates $\mathrm{P}<0.05$, which is meaningful.

Table3. Comparison of the incidence of adverse reactions between the two groups of patients [n(\%)]

\begin{tabular}{ccccc}
\hline Group & Drug resistance & Rash & Sick and vomit & Incidence \\
\hline $\begin{array}{c}\text { Experimental } \\
\text { group }(\mathbf{n}=\mathbf{5 0})\end{array}$ & $1(2.00)$ & $1(4.00)$ & $0(0.00)$ & $2(4.00)$ \\
$\begin{array}{c}\text { Control group } \\
(\mathbf{n}=\mathbf{5 0})\end{array}$ & $6(6.00)$ & $5(8.00)$ & $4(4.00)$ & $15(30.00)$ \\
$\mathrm{X}^{2}$ & & & & 5.586 \\
$\mathrm{P}$ & & & & $<0.05$ \\
\hline
\end{tabular}

\section{Conclusion}

Pediatric pneumonia mostly occurs in infants and young children. It is affected by factors such as young children and poor physical fitness. After the children are given symptomatic treatment, they usually have a long-term unhealed situation. The clinical symptoms are mainly pulmonary rales, Cough, sputum, etc., which pose a serious threat to the health of children, and not conducive to the growth and development of children, and easily reduce the quality of life of children ${ }^{[4]}$. Western medicine treats children with antibiotics or antitussive drugs. The clinical effects of these drugs are limited, which can easily lead to drug resistance in children. From the perspective of traditional Chinese medicine, it is believed that the main pathogenic factor leading to the onset of pneumonia in children with recovery period is the deficiency of lung and spleen qi ${ }^{[5]}$. To treat it, it is necessary to promote blood circulation and remove blood stasis, or apply external treatment methods. The main purpose of treatment is to use Chinese medicine to regulate the spleen and stomach of the child, realize the function of regulating qi and blood, strengthen the body and eliminate evil wind, and ensure that both the symptoms and the root cause are cured ${ }^{[6]}$. The main ingredients of Zhifei Kangfu Decoction include Fritillaria, Tangerine Red, Red Peony Root, Zedoary Turmeric, Peach Seed, Cabbage Seed, Coltsfoot and Aster, etc., which can relieve cough and resolve phlegm, invigorate the spleen and stomach, regulate qi and promote blood circulation. Among them, coltsfoot and aster have similar application effects. They can treat coughs. Aster uses its roots, while coltsfoot uses its flowers. Coltsfoot is sweet and moisturizing, which can go through the lung meridians and blood. It is not hot, pungent but not dry, sweet but not stagnant which is a good medicine for nourishing the lungs, resolving phlegm and cough, so it has a significant effect in the treatment. While orange peel enters and Chinese stomach medicines, and enters lower qi to eliminate phlegm medicines[7]. Scutellaria has the effect of eliminating bloating, reducing qi and phlegm, and promoting digestion and regulation of qi. When the three drugs are used in combination, it can clear away heat and resolve phlegm, moisturize the lungs and relieve cough, and eliminate food and qi. The symptoms of chronic cough in children are mainly due to poor circulation of Qi and blood, and blood stasis in the patient. On this basis, the application of peach kernel, red peony root, and zedoary can realize the effect of blood circulation, disperse stasis, heat-clearing, blood-cooling and clearing residual heat. Licorice can reconcile various drugs and promote the full play of the efficacy [8]. Therefore, when Zhifei Kangfu Decoction is used, it can effectively regulate the lungs and stomach, treat both the symptoms and the root causes, and the effect is significant. And after it is applied, it will not cause serious adverse reactions in children. After applying it in this study, the results showed that the treatment efficiency of children in the experimental group was higher, which was $96.00 \%$, and the children had less cough, asthma, treatment time, etc., which has a positive significance in promoting the recovery of children. The incidence of adverse reactions in children was $4.00 \%$, which was significantly lower than $30.00 \%$ in children in the control group. The data differed greatly, indicating that Zhifei Kangfu Decoction has a significant application effect in children with pneumonia during the recovery period, and has significant clinical significance.

To sum up, the application of Zhifei Kangfu Decoction in the treatment of children with pneumonia during the recovery period has significant clinical effects, which can improve the children's adverse symptoms, reduce the incidence of adverse reactions in children, and shorten the 
children's treatment time, which is of high clinical application value and can be promoted.

\section{Acknowledgement}

As this article is about to close, I would like to express my sincere gratitude to those who have helped in writing this thesis. Thank you for your thought guidance during the writing of this paper, for your careful guidance during the writing of the paper, and for your careful review when the paper is completed. Secondly, I would like to thank the authors of the relevant materials consulted during the writing of this thesis for providing reference materials for my thesis writing.

\section{Reference}

1. Yang Wenmei. The effect analysis of inquiring nursing model in the treatment of bronchial pneumonia in children[J]. Journal of Qiannan Medical College for Nationalities, 2019, v.32(04):6365 .

2. Wang Wei. Discuss the efficacy of integrated traditional Chinese and western medicine in the treatment of bronchial pneumonia in children[J]. China Practical Medicine, 2020, v.15(16):150-152.

3. Ruan Mengran, Xue Jie, Jin Yue, et al. The effect of lung physiotherapy in the treatment of bronchial pneumonia in children[J]. Digest of World Latest Medical Information (Electronic Edition), 2019, 019(088): 95-96.

4. Li Guangping, Cai Jinyun. The application value of Xiaoerfeike Granules in adjuvant treatment of children with bacterial pneumonia during the recovery period[J]. Inner Mongolia Traditional Chinese Medicine, 2019(5).

5. Ge Min, Tao Ye, Li Hua. Observation on the curative effect of combined internal and external treatment of children with pneumonia in the recovery of lung and spleen Qi deficiency in 40 cases[J]. Journal of External Therapy of Traditional Chinese Medicine, 2019, v.28; No.159(02):22- twenty three.

6. Dong Jiabin. Analysis of the efficacy and safety of pediatric massage therapy in the treatment of bronchial pneumonia in children[J]. Health for All, 2020, No.523(14):319-319.

7. Ma Yanna. Clinical Observation on the Treatment of 64 Cases of Infantile Bronchopneumonia with Western Medicine and Qingfei Huatan Decoction[J]. Chinese National Folk Medicine, 2019, v.28; No.345(04):108-109+123.

8. Bai Dongxue. The clinical efficacy of Dingchuan Decoction in the treatment of bronchial pneumonia in children[J]. Diet Health Care, 2019, 6(002):90-90. 